\title{
Correction to: Impacts of Automation Reliability and Failure Modes on Operators' Performance in Security Screening
}

\author{
Zijian Yin, Pei-Luen Patrick Rau, and Zhizhong Li
}

Correction to:

Chapter "Impacts of Automation Reliability and Failure Modes on Operators' Performance in Security Screening" in: D. Harris (Ed.): Engineering Psychology and Cognitive Ergonomics, LNAI 11571, https://doi.org/10.1007/978-3-030-22507-0_11

In the original version of this chapter Reference 6 was published incorrectly. Reference 6 has now been corrected. 